\title{
Analysis of Quality Safety of Agro-products Influenced by Farmers' Production Behaviors
}

\author{
Wanglin Ma (Corresponding author) \\ School of Economics and Management, Sichuan Agricultural University \\ Chengdu, China \\ E-mail: mawanglin94@sina.com \\ Dating Ren \\ School of Economics and Management, Sichuan Agricultural University \\ Chengdu, China \\ E-mail: rdt123@263.net
}

Received: May 3, 2011 Accepted: May 19, 2011 doi:10.5539/jas.v3n3p167

This research is supported by the project of "Research on Safety Supply System of Agro-Products" of Sichuan Province Office of Education humanities and social science research foundation "211"project under (No. CR2110924)

\begin{abstract}
Farmers' production behaviors were important factors which caused quality safety problems of agro-products. Based on the survey in parts of four provinces of Sichuan, Gansu, Henan and Hubei, from the perspective of behaviors of production information acquisition and decision-making behaviors of material inputs, this paper analyzed quality safety of agro-products influenced by farmers' production behaviors in production process. After the analysis, this paper discussed the countermeasures that could guarantee the quality safety of agro-products.
\end{abstract}

Keywords: Behaviors of Production Information Acquisition, Behaviors of Material inputs, Quality Safety of Agro-products

\section{Introduction}

Farmers' production behaviors refer to a general item of a series of activities, which is in certain social environment and in order to satisfy certain goals, that has taken by farmers in the material products production process, mainly including agriculture to produce antenatal, produce medium, postpartum. Farmers' production behaviors are the most crucial man-made factors causing quality safety problems of agricultural products (Zhang $\& \mathrm{Li}, 2004)$. Only by producing agricultural products according to prescribed technical factors and the operating rules, choosing production seed breeds scientifically and reasonably, using agricultural inputs accorded with the national requirements such as pesticide, fertilizer and auxin and so on, can farmers produce safe agricultural products which are meeting the quality standard.

As an agricultural country with a long history, the masses of decentralized management of farmers are still the main bodies in agricultural production at present in China. Farmers' production behaviors directly determine the quality safety level of agricultural products (Wang $\& \mathrm{Xu}, 2004$ ). In agricultural production process, improper use of agricultural inputs such as pesticide, fertilizer and auxin and so on in order to seek the maximum outputs and profits, which seriously influenced the agro-products quality safety. The factors such as lower level of safety production technology, weak safe production consciousness also seriously restricted the farmers' safe production behaviors. Farmers are lacking of understanding on rational use rules and standard production operation specification about agricultural inputs, which urgently needs government technical guidance and services (Feng $\& \mathrm{Li}, 2007)$. Therefore, it has a very important realistic significance to discuss how to standardize farmers' production behavior to ensure agricultural safety in production links and comprehensively enhance the quality safety level of agricultural products in China.

\section{Data sources}

The sample of this study was selected from parts of farmers from four provinces of China including Sichuan, Gansu, Henan and Hubei, mainly based on the agricultural development level and samples of dispersion and random process. Sample data are from investigation in July and August, 2010. By means of interviewing with local farmers and filling out questionnaires, 300 questionnaires in all were given out, of which southern 
provinces and northern provinces(Note 1) were all 150 copies, specific distribution is each 100 copies in Sichuan and Gansu province, each 50 copies in Henan and Hubei province, Finally 297 valid samples were collected and the data analyzed using Excel and Spss statistical software. Farmers' basic situation and the production situation showed in Table 1.

Shown from the investigation results and Table 1: (1) The average age of the interviewed farmers is 45.61 years old; (2) Farmers education level is low, and the junior middle school and the following cultural degree of respondents close to $61.62 \%$; (3) $77.44 \%$ of farmers have family members more than four people; (4) The mean value of family labors is less than 3; (5)Growing agricultural cultivated land area is the least to 0.03 hectare and the largest to 1.33 hectare.

\section{Farmers' Production Behaviors and Agro-products Quality Safety}

Given that the farmers' production behaviors of produce antenatal, produce medium played decisive roles on quality safety of agro-products and with the related investigation, this paper, from two aspects of farmers' behaviors in production information acquisition and decision-making behaviors of material inputs, studied the influence of farmers' behaviors on quality safety of agricultural products, in which farmers' behaviors of production information acquisition were discussed mainly from the acquisition ways of new seed varieties and new production technology, and farmers' decision-making behaviors of material inputs majorly analyzed from use ways of pesticides, chemical fertilizers and auxin(Note 2).

\subsection{Related Behaviors of Production Information Acquisition and Quality Safety of Agro-products}

\subsubsection{New Seed Varieties Information Acquisition Ways and Quality Safety of Agro-products}

Disease resistance and adapting to local climate condition are two of important characteristics of new seed varieties. It is obvious that new seed varieties are good for using less pesticides and producing quality safety of agro-products. What's more, new seed varieties also have many other characteristics such as high quality, high yield, good storage, good commodity nature and adaption to the market, etc. In 2010, southwest provinces of China suffered drought, but corn demonstration variety with planting areas 34.4 hectare called Yunduan 88 won high-yielding in Lufeng county, Yunnan province (one of those southwest provinces)(Note 3), which is also conformed the importance of promoting agricultural new seed variety. However, information impeded is an important factor which affects new seed varieties' inputs, resulting in the limitations of planting area of new seed varieties, and then influencing safe agricultural products production.

Results showed that relatives and neighbors, agricultural technology department and media such as broadcast and television are the main channels used by farmers of getting new seed varieties information, which have percentage of responses (Note 4) respectively $44.1 \%, 31.6 \%$ and $10.2 \%$ (showed in Table 2), indicating that the relatives and neighbors play the most important part in the process of conveying new seed varieties information. Intermediary service organizations and professional associations' functions are very limited and their percentage of responses are $5.3 \%$ and $1.9 \%$ respectively, mainly because the intermediary service organizations and professional association usually charge a fee, farmers generally rarely consult. Some farmers are willing to choose the new seed varieties of agricultural products on their own terms with its percentage of response $7 \%$.

Depth survey showed that any fault in new seed varieties selection probably influenced normal production because of farmers' lack of market information and weak production capacity and all efforts would be in vain, which caused their behaviors outward-looking and herding. Once found the new seed varieties information received from the relatives and neighbors was wrong, but that wrong production information would likely be transmitted perniciously and widely. Thus, agricultural science and technology departments should play active roles to help farmers' selection of improved seed varieties by propagandizing, educating and guiding in the countryside, and then promote safe production of agro-products.

\subsubsection{New Technology Information Acquisition Ways and Quality Safety of Agro-products}

Along with diversified trend development of the agricultural production methods, new production technology can provide more scientific guiding basis for producing safe agricultural products, for instance, spraying techniques of pesticides production provides. For example, development backwardness of the spraying technology of pesticides is the main reason of aggravating negative affect of pesticide use, also one of main reasons affecting the quality safety of agricultural products. However, the new spraying technology of pesticides can better control diseases and insect pests in agricultural production according to different crops and different spraying period, which will help scientifically and reasonably produce quality safety agricultural products.

Results showed that relatives and neighbors and agricultural technology department are main channels used by farmers to get new technology information of agricultural products and their percentage of responses respectively are $38.8 \%$ and $32.5 \%$ (showed in Table 3). Second is to explore the technology according to 
production practice with the percentage of response $10.8 \%$. The functions of the promotion of newspapers, magazines and television, intermediary service organizations, and professional associations are not obvious with the percentage of responses respectively $10 \%, 5.8 \%$ and $2.1 \%$, and depth investigation showed that because the farmers rarely pay close attention to media, in addition, profitability of intermediary service organizations and professional associations caused the farmers got very little information there.

In 2010, the average wheat yields with a planting area 2000 hectare was the highest with 9174 kilograms per hectare in agricultural demonstration garden in Hebi city, Henan province(Note 5); the average rice yields is reached 10729 kilogram per hectare in Dapo demonstration garden in Zhongshu town, Yunnan province(Note 6). These achievements stated that agricultural production technology is important in modern agriculture development. Farmers ate the subjects of agricultural production, only by altering the management way, changing the traditional planting technology and continuously learning the practical production technology, can environmental agricultural products with quality safety, high efficiency and low pollution be produced. If the agricultural manufacturing technology level is backward and the farmers cannot use the agricultural inputs such as pesticides and chemical fertilizers scientifically and reasonably like pesticide match against the proportion, chemical fertilizer use beyond control and unable to use appropriate chemical fertilizer seed varieties, etc, which will affect the quality safety of agricultural products. Therefore, it is necessary for the relevant departments to do more efforts to promote the new technology of agriculture production and guide the farmers better for agricultural production.

\subsection{Related Behaviors of Material inputs and Quality Safety of Agro-products}

Agricultural inputs (including pesticides, fertilizers, agricultural auxins, etc) are key factors to influence quality safety of agricultural products in the production links. Improper use of agricultural inputs such as pesticides and chemical fertilizers in the agricultural production process causes chemical reagent residual beyond bid in agricultural products, which has become one of major problems of quality safety of agricultural products. As the widespread promotion and use of the agricultural inputs like pesticides and chemical fertilizers helps to increase the agricultural outputs substantially, also brings hidden trouble of quality safety of agricultural products. Long-term, especially excessive use of those agricultural inputs will not only pollute the environment in producing areas, but also do harm to human beings because of chemical pesticide residual. This paper started with the inputs of pesticides, chemical fertilizers and auxin which are the most important factors influencing safe production of agricultural products, analyzing the influences of farmers' decision behaviors in the producing process to quality safety of agricultural products.

\subsubsection{Pesticide Use Ways and Quality Safety of Agro-products}

Pesticide was an important input factor used to control the major crop diseases and guarantee agricultural harvests. But pesticide was a double-edged sword, on the one hand, it could ensure quantity safety in agricultural production, for instance, only the pesticide could control the harm when the crop disease and pest happened in a large area, on the other hand, the pesticide caused unsafe agricultural products because of its toxicity if used unreasonably and unscientifically and caused negative effects. What is more, pesticide was a main factor which influenced quality safety of agricultural products because of seed varieties and widely use.

Survey results showed that, using pesticide according to pest existing condition is the leading way used by farmers with the percentage of response $40.1 \%$, the main reason is that the farmers can not distinguish some new crop disease but accept the suggestions given by staffs in pesticide stores according to their description. $27.1 \%$ and $19.0 \%$ of percentage of responses respectively shows the frequency of ways using pesticides according to past experience and specification requirements. Some parts of farmers use the pesticides by consulting their relatives and neighbors. Only $2.6 \%$ of farmers use pesticides at their own will. Told by the $129.3 \%$ of percentage of cases (Note 7), the farmers probably decide the use information and use level of pesticide by one or more ways which indicate their cautions in the process of pesticide use.

Through survey knowledge further, the farmers would increase the dosage of pesticide without considering more and increase the pesticide spraying times in order to improve control effect when the crops become weak resistant, and the farmers probably mixed some kinds of pesticides according past experience to use when the plant diseases and insect pests were turned out to be serious. But, abuse and wrong use of pesticides caused that pesticides residues in agricultural products exceeded maximum residue limit. The price of pollution-free biological pesticides was relatively high, so the farmers would use some pesticide with high residues but lower price to decrease the cost(Sun, 2008), these pesticides had been usually banned by explicit order, for instance, benzene hexachloride, dichloro-diphenyl-tricgloroethane, toxaphene, parathion-methyl and moncrotophos, and the pesticide shopkeeper would like to meet the farmers demand at full stretch in order to make its profit maximization, which all influenced agricultural products safety seriously. 


\subsubsection{Chemical Fertilizer Use Ways and Quality Safety of Agro-products}

The use of chemical fertilizer is effective to improve agricultural yields, but, excessive fertilization will increase the soil acidity, improve soil electrical conductivity, change aggregation degree of nitrate and potassium nitrate and cause soil acidification and salty. What's more, the use of chemical fertilizer will bring some disadvantages like soil nutrient elements unbalance and soil compaction.

Survey results showed that soil fertilizer helped the farmers to decide the chemical fertilizer seed varieties to buy and use level mainly based on the accumulated production experience with the percentage of response $36.39 \%$. The two ways according to the past experience and promoting information were followed with percentage of responses respectively $31.0 \%$ and $13.1 \%$. The percentage of response $(8.2 \%)$ of consulting relatives and neighbors indicated that the way of using chemical fertilizer was not active (shown in Table 5). Judging from the percentage of cases $(131.3 \%)$, more than one way could be adopted by farmers when the chemical fertilizer was used.

Known from the farmers' feedback information, they always judge the soil fertilizer according to the last crops' growing quality. If the last crop growing quality was satisfactory and relative little fertilizers will be used, otherwise, more fertilizer will be used. Using chemical fertilizer according to past experience is also a main way for decision-making about fertilizer use, it has cross-sector with the one mentioned above. Those two ways are all lack of scientificity and bad for safe production of agricultural products.

\subsubsection{Auxin Use Ways and Quality Safety of Agro-products}

In agricultural production process, the use of auxin can significantly promote crop growth, induce its growth to the most favorable direction, maintain physiological action like apical dominance, restrain the flowering of short-day plants and promote the flowering of long-day plants. Currently, the growth hormone is the most common used auxins for the crops in the market, such as indoles compounds including 3 - indoles acetic acid (IAA), indoles propionic acid, indoles butyrate and indolethylamine, etc; Naphthalene compounds including naphthalene acetic acid (NAA), naphthalene propionic acid, naphthalene acetamide, naphthalene methyl acetate, naphthalene butyrate and naphthoxyacetic acid, etc; phenol compounds including para-chlorophenylalanine(PCP), p-lodophenoxyacetic acid, 2-methyl-4-chloro-phenoxyacetic acid, phenoxyacetic acid and phenylacetic acid, etc. Rational use of auxins is beneficial to produce safe agricultural products.

From the survey results, it is easy to know that 86 of all interviewed farmers never used the auxin mentioned above. From those 211 farmers who used auxin, most of them used the auxin mainly according to the requirements of the specifications with the percentage of response $45.5 \%$, some farmers used the auxin according to characteristics of agricultural production and suggestions by the relatives and neighbors with percentage of responses respectively $21.4 \%$ and $18.6 \%$, and farmers seldom used the auxin at their own will which can be known from the percentage of response $2.0 \%$ (shown in Table 6 ).

Through the further understanding, some farmers never used agricultural auxin, mainly because they didn't know the auxin at all. In view of the above analysis, hoping that the agricultural extension department can promptly shift work mode, enhance the farmers' cognition to agricultural auxins by propagandizing, guide the farmers in reasonable way to use the auxins in correct way and then promote safe production of agricultural products.

\section{Conclusions}

In agricultural production process, the farmers' production behaviors directly influence the quality safety of agricultural products. The getting channels of new seed varieties information and new technology information about agricultural products and decision-making behaviors of production factors inputs such as chemical fertilizers, pesticides and auxin have direct influences on quality safety of agricultural products, the specific performances are: (1) The farmers are not very understanding the market information when they are getting the information about new seed varieties and new technology of agricultural products, the relative information received from relatives and neighbors is not scientific, the professional associations' function is not obvious; (2) In certain areas, it is convenient to buy the pesticide according to their own experiences or suggestions given by the staffs in pesticide stores, but contains certain blindness; (3) It is not scientific and normative to use the chemical fertilizers according to past experiences and bad for crops' growth, then influence the quality safety of agricultural products; (4) Farmers are seriously lack of understanding about auxins, some farmers never use auxins or use them in improper way.

\section{Countermeasures}

Based on the above analysis, to guarantee the quality safety of agricultural products in china, following suggestions were put forward in this paper: (1) Increasing the promotion efficiency of agricultural technology 
extension department in new seed varieties and new technology of agricultural production. The government should make greater efforts to propagandize the information about new seed varieties and new technology by the local village committee and the local media, improve the farmers' cognition ability and adoption positivity of new seed varieties and new technology, help to increase the farmers' incomes and promote the healthy development of the local agriculture. (2) Strengthening the supervision on agricultural inputs. The safe production and use of agricultural inputs such as pesticides, chemical fertilizer and auxins directly influenced the quality safety of agricultural products, so the government and the related department should strengthen the regulation and supervision to agricultural inputs and guarantee safe production of agricultural products on the basis of ensuring security supply of agricultural inputs. (3) Standardizing farmers' production behaviors. It is necessary to enhance farmers' use level of pesticide, chemical fertilizer and auxin in agricultural production process, especially auxin use level, by some measures like training and propagandizing, therefore guide farmers' decision-making behaviors of agricultural production scientifically and reasonably and promote safe production of China's agricultural products.

\section{Acknowledgement}

The authors are grateful to Min Wu, Ruiqing Qiao, Jiqiu Zhang \& Ling Li for data collection and Zeng Lin for his valuable suggestions.

\section{References}

Dou, Yanfen, Chen, Tong \& Liu, Lin. (2009). Study on Problems of Quality Safety of Agro-products Based on Agricultural Production Processes. Journal of Tianjing Agricultural University, No. 3. pp: 52-55.

Feng, Zhongze \& Li, Qingjiang. (2007). Analysis of Farmers' Cognition of Quality Safety of Agricultural Products and Influence Factors. Journal of Agricultural Economics, No4. pp:22-25.

Hou Bo, Hou Jing \& Wang Zhiwei. (2010). Farmer's Perception of Pesticide Residue and Its Influence on Behavior Pesticide Application. Heilongjiang Agricultural Sciences, No.2.

Lu, Min \& Li, Yu. (2010). Analysis of Production Information of Edible Fungi and relevant Decision-making Behaviors from Farmers' Perspective Journal of Agrotechnical Economics, No.4. pp:107-113.

$\mathrm{Pu}$, Xujin, Wu, Linhai \& Cao, Wenbin. (2010). Research on a Self-restrict Mechanism of Farmers'Behavior of Utilizing Pesticides: Proto-institutions, Community Norms \& Equilibrium. Science \& Technology and Economy, No.1.

Sun, Qingzhen. (2008). Analysis on the Causal Factors of Household Agro-products Safety. Food and Nutrition in China, No.1. pp:15-17.

Wang, Huashu \& Xu, Xiang. (2004). Micro-behavior and Safety of Agro-products: an Analysis of Rural Household' Production and Residents' Consumption. Journal of Nanjing Agricultural University, No.4. pp:23-28.

Yang Helian, Zhang Jianwei, Sun Li, etc. (2010). Investigation on Pesticides Application of Vegetable Farmers in Xinxiang, Guizhou Agricultural Sciences, No.1.

Yang jian, Gao Shenrong \& Wu Linhai. (2010). Research on Pesticide Application Behavior of Scattered Farmer. Heilongjiang Agricultural Sciences, No.1.

Yang, Jian, Hou, Bo \& Wu Linhai. (2010). Summarize of the Negative Effects of Chemical Pesticides, Farmers Pesticide Application Practices and Influencing Factors. Guangdong Agricultural Sciences, No.3.

Zhang, Yaogang \& Li Gongkui. (2004). Consequence analysis of agricultural products quality and safety by farmer. Journal of Productive Research, No.6:34-35.

Zhu yin \& Wu, Linhai. (2010). Comparision analysis of Farmers pesticide using ways with different planting areas. Journal of Zhejiang Agricultural Science, No.5.

\section{Notes}

Note 1. In this paper southern provinces refer to Sichuan and Hubei and northern provinces refer to Gansu and Henan.

Note 2. In this paper, the agricultural auxins mainly refer to agricultural auxin.

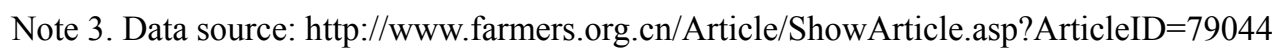

Note 4. Percentage of response refers to the percentage of response times of each option to response times of all options. In this paper, it represents a proportion of the farmers' behavior in choosing some decisions (reflected by the answering times) to all decision-making behaviors (reflected by all answering times), the greater the proportion, the bigger the tendency the way chosen by the farmers. 
Note 5. Data source: http://finance.sina.com.cn/roll/20100617/14438127876.shtml

Note 6. Data source: http://www.yn.xinhuanet.com/newscenter/2010-10/18/content_21154330.htm

Note 7. Percentage of cases refers to the percentage of response times of each option to valid sample numbers. In this paper, it represents a proportion of the farmers' behavior in choosing some decisions (reflected by the answering times) to the total sample numbers, the greater the proportion, the bigger the mixed ways of farmers' decision-making behaviors, which means that the bigger the proportion, the farmers would probably choose several decision-making ways in agricultural production.

Table 1. Statistical Description of Sample Farmers' Basic Situation

\begin{tabular}{|l|c|c|c|c|}
\hline & Max. & Min. & Mean & Standard Deviation \\
\hline Age (in years) & 20 & 77 & 45.61 & 10.77 \\
\hline Education (in years) & 0 & 16 & 6.92 & 3.62 \\
\hline Family size (in persons) & 1 & 8 & 4.58 & 1.46 \\
\hline Family labors size (in persons) & 1 & 4 & 2.45 & 0.85 \\
\hline Total area under cultivation (Mu) & 0.5 & 20 & 5.53 & 3.57 \\
\hline
\end{tabular}

Table 2. New Seed Varieties Information Acquisition Ways

\begin{tabular}{|l|c|c|}
\hline & Percentage of responses(\%) & Percentage of cases (\%) \\
\hline Relatives and neighbors & 44.1 & 55.6 \\
\hline Agricultural technology department & 31.6 & 39.7 \\
\hline Media such as broadcast and television & 10.2 & 12.8 \\
\hline Determining by themselves & 7.0 & 8.8 \\
\hline Intermediary service organizations & 5.3 & 6.7 \\
\hline Professional associations & 1.9 & 2.4 \\
\hline \multicolumn{1}{|c|}{ Total } & 100.0 & 125.9 \\
\hline
\end{tabular}

Table 3. New Technology Information Acquisition Ways

\begin{tabular}{|l|c|c|}
\hline & Percentage of responses(\%) & Percentage of cases(\%) \\
\hline Relatives and neighbors & 38.8 & 49.5 \\
\hline Agricultural technology department & 32.5 & 41.4 \\
\hline Self grope & 10.8 & 13.8 \\
\hline newspapers, magazines and television & 10 & 12.8 \\
\hline Intermediary service organizations & 5.8 & 7.4 \\
\hline professional associations & 2.1 & 2.7 \\
\hline \multicolumn{1}{|c|}{ Total } & 100.0 & 127.6 \\
\hline
\end{tabular}

Table 4. Pesticides Use Ways

\begin{tabular}{|l|c|c|}
\hline & Percentage of responses(\%) & Percentage of cases(\%) \\
\hline According to pest existing condition & 40.1 & 51.9 \\
\hline According to past experience & 27.1 & 35.0 \\
\hline According to specification requirements & 19.0 & 24.6 \\
\hline Consulting relatives and neighbors & 11.2 & 14.5 \\
\hline At his/her own will & 2.6 & 3.4 \\
\hline Total & 100.0 & 129.3 \\
\hline
\end{tabular}

Table 5. Chemical Fertilizer Use Ways

\begin{tabular}{|c|c|c|}
\hline & Percentage of responses( $(\%)$ & Percentage of cases $(\%)$ \\
\hline According to the soil fertility & 36.9 & 48.5 \\
\hline According to past experience & 31.0 & 40.7 \\
\hline According to promoting information & 13.1 & 17.2 \\
\hline At his/her own will & 10.8 & 14.1 \\
\hline Consulting relatives and neighbors & 8.2 & 10.8 \\
\hline Total & 100.0 & 131.3 \\
\hline
\end{tabular}

Table 6. Auxin Use Ways

\begin{tabular}{|l|c|c|}
\hline & Percentage of responses(\%) & Percentage of cases(\%) \\
\hline According to specification requirements & 45.5 & 47.4 \\
\hline $\begin{array}{l}\text { According to characteristics of } \\
\text { agricultural production }\end{array}$ & 21.4 & 22.3 \\
\hline Consulting relatives and neighbors & 18.6 & 19.4 \\
\hline According to past experiences & 11.8 & 12.3 \\
\hline At his/her own will & 2.7 & 2.8 \\
\hline \multicolumn{1}{|c|}{ Total } & 100.0 & 104.0 \\
\hline
\end{tabular}

